COMMUNICATIONS IN

ANALYSIS AND GEOMETRY

Volume 4, Number 3, 459-480, 1996

\title{
Geometric Expansion of Starshaped Plane Curves
}

\author{
DoNG-Ho TSAI
}

\section{Introduction and main result.}

In this paper we shall deal with general nonhomogeneous expanding curvature flows of an arbitrary starshaped closed plane curve $\gamma_{0}$ in $\mathbf{R}^{2}$. This is a partial generalization of Chow-Tsai [5] for nonhomogeneous expanding curvature flows of closed convex plane curves. A major difference between these two papers is that in Chow-Tsai [5] we use the support functions of convex closed curves to formulate our problem while we use the radial functions of starshaped closed curves here. In a later paper by Chow-LiouTsai [8], we shall generalize our results to the expansion of embedded plane curves with turning angle greater than $-\pi$. Although we concentrate on nonhomogeneous expanding curvature flows here, we should mention the many important works on homogeneous curvature flows of closed convex curves and hypersurfaces in the last several years. We refer the readers to the introductory remarks in the papers by Andrews [1], Chow-Tsai [5], or Chow-Liou-Tsai [7] for further information. For expanding starshaped hypersurfaces, we mention the papers by Urbas [19] and Gerhardt [10], where they consider the homogeneous expanding curvature flows of starshaped hypersurfaces with dimension at least two. Recently, Chow [2] was able to use the Aleksandrov reflection method to get a gradient estimate for the radial functions of nonhomogeneous expanding curvature flows of starshaped hypersurfaces.

Let $\gamma_{0}$ be an arbitrary starshaped (with respect to the origin of $\mathbf{R}^{2}$ ) embedded closed curve parameterized by a smooth embedding $X_{0}(u): S^{1} \rightarrow$ $\mathbf{R}^{2}$. We consider the initial value problem

$$
\begin{aligned}
& \frac{\partial X}{\partial t}=F(K) \cdot N \\
& X(u, 0)=X_{0}(u)
\end{aligned}
$$

where $F(z): \mathbf{R} \rightarrow \mathbf{R}^{+}$is an arbitrary positive smooth decreasing function which satisfies $\lim _{z \rightarrow-\infty} F(z)=\infty$ and $\dot{F}(z)=\frac{d F(z)}{d z}<0$ everywhere, $K(u, t)$ is 
the curvature of the curve given by $X(\cdot, t)$ at the point $u$, and $N(\cdot, t)$ is the unit outward normal vector field to the curve $X(\cdot, t)$. Note that we only put very little assumptions on $F$. The condition $\dot{F}<0$ will guarantee that our equation (1.1) is parabolic (see (1.2) below), while the condition $\lim _{z \rightarrow-\infty} F(z)=\infty$ is used in the second derivative estimate, it is not required in the gradient estimate. Moreover, when $\lim _{z \rightarrow-\infty} F(z)<\infty$, singularities may develop for certain initial data (see the picture in Chow-Liou-Tsai [8]). Geometrically speaking,equation (1.1) means that we deform the initial curve $\gamma_{0}$ in its outward unit normal direction with arbitrary speed $F$ depending on its curvature $K$ only. Intuitively, we expect the evolving curves $\gamma_{t}$ remain starshaped and expand to infinity while their shapes asymptotically become round. This is indeed the case as we shall show below. It is natural to consider expansion with nonhomogeneous speed when the initial curve $\gamma_{0}$ is starshaped. The reason is that, in this case, homogeneous speed corresponds to the choice $F(K)=\frac{1}{K}$, which no longer makes sense since the curvature may be zero somewhere.

Since $\gamma_{0}$ may not necessarily be convex, we can no longer use the support function on $S^{1}$ to reformulate equation (1.1) (see Urbas [18]). However, by introducing polar coordinates $(r, \theta)$ in $\mathbf{R}^{2}$, equation (1.1) for starshaped plane curves expansion is equivalent to the following

$$
\begin{aligned}
& \frac{\partial r}{\partial t}=\frac{\sqrt{r^{2}+r_{1}^{2}}}{r} \cdot F(K) \\
& r(\theta, 0)=r_{0}(\theta),
\end{aligned}
$$

where $r_{0}(\theta)$ is the radial function of the initial curve $\gamma_{0}, K=\frac{r^{2}+2 r_{1}^{2}-r r_{2}}{A^{3 / 2}}$ is the curvature of the starshaped plane curves, $A=r^{2}+r_{1}^{2}$, and $r_{1}=$ $\frac{\partial r}{\partial \theta}, r_{2}=\frac{\partial^{2} r}{\partial \theta^{2}}, \ldots e t c$. The proof of the equivalence is similar to what Urbas did in his paper [19] for the higher dimensional case. We will not repeat it. Here we should point out that in equation (1.1), the partial derivative $\frac{\partial}{\partial t}$ is with respect to fixed $u$, while in equation (1.2), $\frac{\partial}{\partial t}$ is with respect to fixed angle $\theta$. To avoid confusion, we use the coordinate $(u, \tau)$ for equation (1.1) and use the coordinate $(\theta, t)$ for equation (1.2) with the understanding that $t=\tau$. In the rest of this paper, we also use ' and "to denote $\frac{\partial}{\partial \theta}$ and $\frac{\partial^{2}}{\partial \theta^{2}}$ respectively when we deal with evolution equations resulting from (1.2).

It is well-known that there exists a unique smooth solution to equation (1.2) (or (1.1)) on $S^{1} \times[0, T)$ for some maximal time $T>0$. We shall prove that the solution exists for long time and show that the evolving curves $\gamma_{t}$, under an appropriate rescaling, converge to the unit circle $S^{1}$ in the sense of 
the following theorem. The maximum principle will be the main ingredient in the proof.

The main result of this paper is the following

Theorem 1.1. Let $F(z): \mathbb{R} \rightarrow \mathbb{R}^{+}$be an arbitrary positive smooth decreasing function with $\dot{F}<0$ everywhere and $\lim _{z \rightarrow-\infty} F(z)=\infty$. For any given smooth positive function $r_{0}(\theta): S^{1} \rightarrow \mathbb{R}^{+}$, there exists a unique solution $r \in C^{\infty}\left(S^{1} \times[0, \infty)\right)$ to equation (1.2) such that $\lim _{t \rightarrow \infty} r_{\min }(t)=\infty$. Moreover, there exists a constant $C$ depending only on $F$ and $r_{0}$ such that

$$
\left|\frac{\partial r}{\partial \theta}(\theta, t)\right| \leq C
$$

for all $(\theta, t) \in S^{1} \times[0, \infty)$. As a consequence, there exists a solution $R(t)$ to the o.d.e.

$$
\frac{d R}{d t}=F\left(\frac{1}{R}\right)
$$

on $[0, \infty)$ such that

$$
r_{\min }(t) \leq R(t) \leq r_{\max }(t)
$$

and the radial functions $\tilde{r}$ of the rescaled curves $\tilde{\gamma}=\frac{\gamma}{R}$ satisfy

$$
\|\tilde{r}(\cdot, t)-1\|_{C^{1}\left(S^{1}\right)} \leq \frac{2 \pi C}{R(t)}
$$

for all $t \in[0, \infty){ }^{1}$

From now on, we shall focus on equation (1.2) and prove the long time existence of a solution. We first compute several evolution equations.

\footnotetext{
${ }^{1}$ Added in proof: In fact, based on the gradient estimate Proposition 3.3 and the curvature estimate Lemma 6.3, we can show that the evolving starshaped curves become convex eventually. As a consequence of that, the radial functions $\tilde{r}$ of the rescaled curves $\tilde{\gamma}=\frac{\gamma}{R}$ will converge to the constant 1 in $C^{2}$-norm. See a later paper by Chow-Liou-Tsai [8].
} 


\section{Preliminary computations and the evolution of curvature.}

Our first lemma is on the growth rate of $r$. Unlike the case in the convex curve expansion where the speed of the support function may blow up in finite time, the speed of $r$ here will stay finite as long as $t$ is finite because of the nature of our problem. We have

Lemma 2.1. Let $r_{\max }(t)=\max _{\theta \in S^{1}} r(\theta, t)$ and $r_{\min }(t)=\min _{\theta \in S^{1}} r(\theta, t)$. We have

$$
a t+r_{\min }(0) \leq r_{\min }(t) \leq r(\theta, t) \leq r_{\max }(t) \leq b t+r_{\max }(0)
$$

for all $(\theta, t) \in S^{1} \times[0, T)$, where $a=F\left(\frac{1}{r_{\min }(0)}\right), b=F(0)$.

Proof. We will use a version of the maximum principle due to Hamilton [12]. Since $r$ is smooth, $r_{\max }(t)$ and $r_{\min }(t)$ are clearly Lipschitz functions of $t$. Therefore

$$
\begin{aligned}
\frac{d^{+}}{d t} r_{\min }(t) & =\lim _{h \rightarrow 0^{+}} \inf \frac{r_{\min }(t+h)-r_{\min }(t)}{h} \\
& \geq \inf \left\{\frac{\partial}{\partial t} r(\xi, t), \xi \in m(t)\right\},
\end{aligned}
$$

where $m(t)=\left\{\theta \in S^{1}: r(\theta, t)=r_{\min }(t)\right\}$. For any $\xi \in m(t)$, we have

$$
K(\xi, t)=\frac{r^{2}-r r_{2}}{r^{3}}(\xi, t) \leq \frac{1}{r}(\xi, t)=\frac{1}{r_{\min }(t)} .
$$

Therefore, since $r_{\min }(t)$ is increasing and $F$ is decreasing, we obtain

$$
\frac{d^{+}}{d t} r_{\min }(t) \geq F\left(\frac{1}{r_{\min }(t)}\right) \geq F\left(\frac{1}{r_{\min }(0)}\right) \text {. }
$$

This implies that

$$
r(\theta, t) \geq r_{\min }(t) \geq a t+r_{\min }(0),
$$

where $a=F\left(\frac{1}{r_{\min }(0)}\right)$. Similarly, we can show

$$
\frac{d^{+}}{d t} r_{\max }(t) \leq F\left(\frac{1}{r_{\max }(t)}\right) \leq F(0)
$$

which yields

$$
r(\theta, t) \leq r_{\max }(t) \leq b t+r_{\max }(0),
$$

where $b=F(0)$. The proof of (2.1) is done.

The evolution of the gradient of $r$ is given by the following 
Lemma 2.2. (i)

$$
\begin{aligned}
\partial_{t} r_{1}= & -\frac{\dot{F}}{A} r_{3}+\left[\frac{F}{A^{1 / 2}}+\frac{\dot{F}}{A^{2}}\left(3 r^{2}-3 r_{1}^{2}+3 r r_{2}\right)\right] \frac{r_{1}}{r} r_{2} \\
& -\frac{F}{A^{1 / 2}} \frac{r_{1}^{3}}{r^{2}}-\frac{\dot{F}}{A^{2}}\left(4 r_{1}^{3}+r^{2} r_{1}\right)
\end{aligned}
$$

on $S^{1} \times[0, T)$, where $r_{3}=\frac{\partial^{3} r}{\partial \theta^{3}}, F=F(K), \dot{F}=\dot{F}(K)=\left.\frac{d F}{d z}\right|_{z=K}$.

(ii)

$$
\begin{aligned}
\partial_{t} w= & -\frac{\dot{F}}{A} w^{\prime \prime}+\left[\frac{F}{A^{1 / 2}}+\frac{\dot{F}}{A^{2}}\left(3 r^{2}-3 r_{1}^{2}+3 r r_{2}\right)\right] \frac{r_{1}}{r} w^{\prime} \\
& -2 \frac{F}{A^{1 / 2}} \frac{w^{2}}{r^{2}}-2 \frac{\dot{F}}{A^{2}}\left(4 r_{1}^{2}+r^{2}\right) w+2 \frac{\dot{F}}{A} r_{2}^{2}
\end{aligned}
$$

on $S^{1} \times[0, T)$, where $w=r_{1}^{2}, w^{\prime}=\frac{\partial w}{\partial \theta}, w^{\prime \prime}=\frac{\partial^{2} w}{\partial \theta^{2}}$.

Proof. The proof is a routine computation. First we have

$$
\partial_{t} r_{1}=\left(\frac{\sqrt{A}}{r} F(K)\right)^{\prime}=\left(\frac{\sqrt{A}}{r}\right)^{\prime} F(K)+\frac{\sqrt{A}}{r} \dot{F} K^{\prime} .
$$

We compute

$$
K^{\prime}=\frac{1}{A^{5 / 2}}\left(-A r r_{3}-A r r_{1}-3 r r_{1}^{3}-3 r_{1}^{3} r_{2}+3 r^{2} r_{1} r_{2}+3 r r_{1} r_{2}^{2}\right)
$$

and

$$
\left(\frac{\sqrt{A}}{r}\right)^{\prime}=\frac{r r_{1}+r_{1} r_{2}}{r A^{1 / 2}}-A^{1 / 2} \frac{r_{1}}{r^{2}} .
$$

Formula (2.2) follows by substituting (2.5) and (2.6) into (2.4). To prove (2.3), we use

$$
w^{\prime}=2 r_{1} r_{2}, \quad w^{\prime \prime}=2 r_{1} r_{3}+2 r_{2}^{2}
$$

and (2.2).

As a preliminary estimate, we have the following lemma, which will be improved later. 
Lemma 2.3. There is a constant $C_{1}$ depending on the initial curve $\gamma_{0}$ and F such that

$$
\frac{r_{1}^{2}(\theta, t)}{r(\theta, t)} \leq C_{1} \quad \text { on } S^{1} \times[0, T)
$$

Proof. Same as before, we have $w=r_{1}^{2}$ and

$$
\begin{aligned}
\frac{d^{+}}{d t} w_{\max }(t) & =\lim _{h \rightarrow 0^{+}} \sup \frac{w_{\max }(t+h)-w_{\max }(t)}{h} \\
& \leq \sup \left\{\frac{\partial}{\partial t} w(\eta, t), \eta \in W(t)\right\},
\end{aligned}
$$

where $W(t)=\left\{\theta \in S^{1}: w(\theta, t)=w_{\max }(t)\right\}$. For any $\eta \in W(t)$, we have $r_{2}(\eta, t)=0$ and

$$
0<K(\eta, t)=\frac{r^{2}+2 r_{1}^{2}}{A^{3 / 2}}(\eta, t) \leq \frac{2}{A^{1 / 2}}(\eta, t) \leq \frac{2}{r_{\min }(t)} \leq \frac{2}{r_{\min }(0)} .
$$

By (2.3), we get

$$
\frac{d^{+}}{d t} w_{\max }(t) \leq-8 \dot{F}(K) \text { at }(\eta, t) .
$$

Let $d=\max |\dot{F}(z)|$ on the interval $\left[0, \frac{2}{r_{\min }(0)}\right]$. We obtain

$$
r_{1}^{2}(\theta, t) \leq w_{\max }(t) \leq 8 d t+w_{\max }(0) .
$$

and conclude

$$
\frac{r_{1}^{2}(\theta, t)}{r(\theta, t)} \leq \frac{8 d t+w_{\max }(0)}{a t+r_{\min }(0)} \leq C_{1}
$$

for all $(\theta, t) \in S^{1} \times[0, T)$, where $C_{1}=\max \left(\frac{8 d}{a}, \frac{w_{\max }(0)}{r_{\min }(0)}\right)$. This finishes the proof of the lemma.

The next lemma, though similar to Lemma 2.3, may be of interest on its own.

Lemma 2.4. Let $(0, \lambda]$ be the maximum interval on which $\dot{F}(z)+\frac{F(z)}{z} \geq$ 0 . For any initial curve $\gamma_{0}$ with radial function $r_{0}(\theta) \geq \frac{1}{\lambda}$, for all $\theta \in S^{z}$, we have

$$
\max _{\theta \in S^{1}} \frac{r_{1}^{2}(\theta, t)}{r^{2}(\theta, t)} \leq \max _{\theta \in S^{1}} \frac{r_{1}^{2}(\theta, 0)}{r^{2}(\theta, 0)} \quad \text { for all } t \in[0, T) .
$$

In particular, if $\dot{F}(z)+\frac{F(z)}{z} \geq 0$ on $(0, \infty)$, then (2.9) holds for any initial curve $\gamma_{0}$. 
Proof. Since this lemma will not be used later, we only provide a brief proof. For convenience, let $\psi=\frac{r_{1}^{2}}{r^{2}}=\frac{w}{r^{2}}$. At any point $(p, t)$ where $\psi$ attains its maximum on $S^{1}$, that is $\psi(p, t)=\max _{\theta \in S^{1}} \psi(\theta, t)$, we have

$$
r_{2}=\frac{r_{1}^{2}}{r} \quad \text { at }(p, t)
$$

This will imply

$$
\begin{aligned}
\partial_{t} \psi(p, t)= & -\frac{\dot{F}}{A} \psi^{\prime \prime}+\left[\frac{F}{A^{1 / 2}}+\frac{\dot{F}}{A^{2}}\left(3 r^{2}-3 r_{1}^{2}+3 r r_{2}\right)\right] \frac{r_{1}}{r} \psi^{\prime} \\
& -2 \frac{r_{1}^{2}}{r^{4}}\left(\dot{F}(K)+A^{1 / 2} F(K)\right) \text { at }(p, t) .
\end{aligned}
$$

Note that

$$
0<K(p, t)=\frac{1}{A^{1 / 2}}(p, t) \leq \frac{1}{r_{\min }(t)} \leq \frac{1}{\min _{\theta \in S^{1}} r_{0}(\theta)} \leq \lambda .
$$

By the assumption, we deduce

$$
-2 \frac{r_{1}^{2}}{r^{4}}\left(\dot{F}(K)+A^{1 / 2} F(K)\right) \leq 0 \quad \text { at }(p, t) .
$$

Hence

$$
\partial_{t} \psi \leq 0 \quad \text { at }(p, t) .
$$

By the maximum principle, (2.9) is proved.

Remark: We would like to point out that the border case of the differential inequality $\dot{F}(z)+\frac{F(z)}{z} \geq 0$ happens to be the homogeneous speed $F(z)=\frac{1}{z}$.

The next lemma will be our main formula, which is the evolution equation of the curvature $K$. When we compute the evolution equation of the curvature $K$ using the coordinate $(u, \tau)$ or arc length parameter $s$, part of our computations are similar to those in the paper by Gage and Hamilton [9]. Details can be found there.

Lemma 2.5. We have

$$
\partial_{t} K=-\frac{\dot{F}}{A} K^{\prime \prime}+\frac{F}{A^{1 / 2}} \frac{r_{1}}{r} K^{\prime}+\frac{A^{\prime}}{2 A^{2}} \dot{F} K^{\prime}-\frac{\ddot{F}}{A} K^{\prime 2}-F K^{2}
$$

on $S^{1} \times[0, T)$, where $\ddot{F}=\ddot{F}(K)=\left.\frac{d^{2} F}{d z^{2}}\right|_{z=K}$. 
Proof. It is possible to give a direct computation of $K$ using $(\theta, t)$ coordinate and the relation $K=\frac{r^{2}+2 r_{1}^{2}-r r_{2}}{A^{3 / 2}}$, but it turns out to be very tedious. We can use a different approach. First, following similar calculations as in Gage and Hamilton [9] and using the $(u, \tau)$ coordinate, we can obtain

$$
\partial_{\tau} K=-\frac{\partial^{2}}{\partial s^{2}} F(K)-F(K) K^{2},
$$

where $s$ denotes arc length parameter. Although $s$ is only defined up to a constant, $\frac{\partial}{\partial s}$ is uniquely defined. Let $X(u, \tau)=(x(u, \tau), y(u, \tau))$. We have the relation

$$
\left(\begin{array}{l}
x(u, \tau) \\
y(u, \tau)
\end{array}\right)=r(\theta(u, \tau), t) \cdot\left(\begin{array}{c}
\cos \theta(u, \tau) \\
\sin \theta(u, \tau)
\end{array}\right) .
$$

Let $P=(\cos \theta(u, \tau), \sin \theta(u, \tau))$ and $J=(-\sin \theta(u, \tau), \cos \theta(u, \tau))$. We deduce the following relation between the two orthonormal frames $\{P, J\},\{N, T\}$ along $\partial_{t}$

$$
\begin{aligned}
& N=\text { unit outward normal }=\frac{1}{\sqrt{A}}\left(r P-r_{1} J\right) \\
& T=\text { unit tangent }=\frac{1}{\sqrt{A}}\left(r J+r_{1} P\right)
\end{aligned}
$$

and

$$
\left(\frac{\partial x}{\partial \tau}, \frac{\partial y}{\partial \tau}\right)=\left(\frac{\partial r}{\partial \theta} \frac{\partial \theta}{\partial \tau}+\frac{\partial r}{\partial t}\right) P+r(\theta, t) \frac{\partial \theta}{\partial \tau} J
$$

Using the equation

$$
\left(\frac{\partial x}{\partial \tau}, \frac{\partial y}{\partial \tau}\right)=F(K) \cdot N=F(K) \cdot \frac{1}{\sqrt{A}}\left(r P-r_{1} J\right),
$$

and comparing (2.12) and (2.13), we can get

$$
\frac{\partial \theta}{\partial \tau}=-\frac{F(K)}{\sqrt{A}} \frac{r_{1}}{r}
$$

We also have

$$
\frac{\partial}{\partial s}=\frac{1}{\sqrt{v}} \frac{\partial}{\partial u}=\frac{1}{\sqrt{A}} \frac{\partial}{\partial \theta}
$$

where

$$
v=\left(\frac{\partial x}{\partial u}\right)^{2}+\left(\frac{\partial y}{\partial u}\right)^{2}
$$


By the relation

$$
K(u, \tau)=K(\theta(u, \tau), t)
$$

we infer

$$
\frac{\partial K}{\partial \tau}=\frac{\partial K}{\partial \theta} \frac{\partial \theta}{\partial \tau}+\frac{\partial K}{\partial t}
$$

It follows that

$$
\begin{aligned}
\frac{\partial K}{\partial t} & =\frac{\partial K}{\partial \tau}-\frac{\partial K}{\partial \theta} \frac{\partial \theta}{\partial \tau} \\
& =-\frac{\partial^{2}}{\partial s^{2}} F(K)-F(K) K^{2}+\frac{\partial K}{\partial \theta} \frac{F(K)}{\sqrt{A}} \frac{r_{1}}{r} \\
& =-\dot{F} K_{s s}-\ddot{F} K_{s}^{2}-F K^{2}+\frac{F}{A^{1 / 2}} \frac{r_{1}}{r} K^{\prime} .
\end{aligned}
$$

Finally, use

$$
\frac{\partial}{\partial s}=\frac{1}{\sqrt{A}} \frac{\partial}{\partial \theta}
$$

to conclude

$$
\partial_{t} K=-\frac{\dot{F}}{A} K^{\prime \prime}+\frac{F}{A^{1 / 2}} \frac{r_{1}}{r} K^{\prime}+\frac{A^{\prime}}{2 A^{2}} \dot{F} K^{\prime}-\frac{\ddot{F}}{A} K^{\prime 2}-F K^{2},
$$

which is equation (2.10).

Lemma 2.5 and the maximum principle will yield the following immediately.

Corollary 2.6. There exists a positive constant $C_{2}$ depending only on the initial curve $\gamma_{0}$ such that

$$
K(\theta, t) \leq C_{2} \quad \text { on } S^{1} \times[0, T) .
$$

Proof. By (2.10), we have

$$
\partial_{t} K \leq-\frac{\dot{F}}{A} K^{\prime \prime}+\frac{F}{r A^{1 / 2}} r_{1} K^{\prime}+\frac{A^{\prime}}{2 A^{2}} \dot{F} K^{\prime}-\frac{\ddot{F}}{A} K^{\prime 2},
$$

since $F$ is positive. Now (2.15) follows from the maximum principle.

Lemma 2.7. The evolution equation of $F(K)$ is

$$
\partial_{t} F(K)=-\frac{\dot{F}}{A} F(K)^{\prime \prime}+\frac{F}{A^{1 / 2}} \frac{r_{1}}{r} F(K)^{\prime}+\frac{A^{\prime}}{2 A^{2}} \dot{F} F(K)^{\prime}-\dot{F} K^{2} F(K)
$$

on $S^{1} \times[0, T)$, where $F(K)^{\prime}=\frac{\partial}{\partial \theta} F(K), F(K)^{\prime \prime}=\frac{\partial^{2}}{\partial \theta^{2}} F(K)$. 
Proof. Clearly, by (2.10), we have

$$
\begin{aligned}
\partial_{t} F(K) & =\dot{F}(K) \partial_{t} K \\
& =\dot{F} \cdot\left(-\frac{\dot{F}}{A} K^{\prime \prime}+\frac{F}{A^{1 / 2}} \frac{r_{1}}{r} K^{\prime}+\frac{A^{\prime}}{2 A^{2}} \dot{F} K^{\prime}-\frac{\ddot{F}}{A} K^{\prime 2}-F K^{2}\right) .
\end{aligned}
$$

Compute

$$
\begin{aligned}
& F(K)^{\prime}=\dot{F} K^{\prime} \\
& F(K)^{\prime \prime}=\dot{F} K^{\prime \prime}+\ddot{F} K^{\prime 2},
\end{aligned}
$$

and use them into (2.17) to get equation (2.16).

\section{Gradient estimate.}

Our main crucial estimate in this section is a uniform bound for the gradient of $r$. Here we use the maximum principle to show that the gradient of $r$ is bounded by a constant $C$ depending on $F$ and the initial curve $\gamma_{0}$. A different important approach was made by Chow recently, where he used the Aleksandrov reflection method to obtain a uniform gradient estimate (with the bound depending only on the initial hypersurface) for the radial function of a starshaped hypersurface moving by its curvature vector, which holds outside some compact set depending only on the initial hypersurface. See Chow [2] for details. Furthermore, the Aleksandrov reflection method has been used in various ways in the papers by Chow-Gulliver [3], [4] recently.

The quantity we shall estimate here is

$$
\frac{Q}{F(K)}, \text { where } Q=\frac{r r_{1}}{\sqrt{A}} .
$$

This quantity is equal to $\frac{r_{1}}{\partial_{t} r}$, the ratio of the space derivative to the time derivative. It is also equal to the ratio of the tangential component of the position vector to the speed function. We shall show that the absolute value of $\frac{Q}{F}$ is bounded by a positive constant depending on $\gamma_{0}$ and $F$. The motivation for estimating this quantity is based on a previous paper. See Chow-Tsai [5].

Remember that we have

$$
\begin{aligned}
& N=\text { unit outward normal }=\frac{1}{\sqrt{A}}\left(r P-r_{1} J\right) \\
& T=\text { unit tangent }=\frac{1}{\sqrt{A}}\left(r J+r_{1} P\right) \\
& X(u, \tau)=r(\theta(u, \tau), t) \cdot(\cos \theta(u, \tau), \sin \theta(u, \tau)) .
\end{aligned}
$$


From this it follows that

$$
\langle X, N\rangle=\frac{r^{2}}{\sqrt{A}},\langle X, T\rangle=\frac{r r_{1}}{\sqrt{A}}
$$

where $\langle\cdot, \cdot\rangle$ denotes the Euclidean inner product.

Lemma 3.1. We have

$$
\text { (i) } \frac{\partial}{\partial \tau}\langle X, N\rangle=-\dot{F}\langle X, N\rangle_{s s}-K^{2} \dot{F}\langle X, N\rangle+F+K \dot{F} \text {. }
$$

$$
\text { (ii) } \frac{\partial}{\partial \tau}\langle X, T\rangle=-\dot{F}\langle X, T\rangle_{s s}-K^{2} \dot{F}\langle X, T\rangle \text {. }
$$

Proof. The Frenet formula says that

$$
\frac{\partial N}{\partial s}=K T, \frac{\partial T}{\partial s}=-K N
$$

We also have the relation for commuting the operators $\frac{\partial}{\partial \tau}$ and $\frac{\partial}{\partial s}$, which is

$$
\frac{\partial}{\partial \tau} \frac{\partial}{\partial s}-\frac{\partial}{\partial s} \frac{\partial}{\partial \tau}=-K F \frac{\partial}{\partial s}
$$

Therefore we can infer

$$
\begin{aligned}
\frac{\partial T}{\partial \tau} & =\frac{\partial}{\partial \tau} \frac{\partial X}{\partial s}=\frac{\partial}{\partial s} \frac{\partial X}{\partial \tau}-K F T \\
& =\frac{\partial}{\partial s}(F N)-K F T=F(K)_{s} N+F K T-K F T \\
& =F(K)_{s} N .
\end{aligned}
$$

Similarly, we have

$$
\frac{\partial N}{\partial \tau}=-F(K)_{s} T
$$

Now by equation (3.5), we find

$$
\frac{\partial}{\partial \tau}\langle X, N\rangle=F-F(K)_{s}\langle X, T\rangle .
$$


Using

$$
\langle X, N\rangle_{s}=\left\langle X, N_{s}\right\rangle=K\langle X, T\rangle
$$

and

$$
\langle X, N\rangle_{s s}=K_{s}\langle X, T\rangle+K+K\langle X,-K N\rangle,
$$

and equation (3.6), we can get (3.1). The proof of (3.2) is similar.

Lemma 3.2. Let $H=\frac{\langle X, T\rangle}{F}=\frac{Q}{F}$. We have

$$
\frac{\partial H}{\partial \tau}=-\dot{F} H_{s s}-2 \frac{\dot{F}}{F} F(K)_{s} H_{s} .
$$

Proof. By equation (3.2), we obtain

$$
\begin{aligned}
\frac{\partial H}{\partial \tau} & =\frac{1}{F}\left[-\dot{F}\langle X, T\rangle_{s s}-K^{2} \dot{F}\langle X, T\rangle\right] \\
& -\frac{1}{F^{2}}\langle X, T\rangle\left[-\dot{F} F(K)_{s s}-\dot{F} F K^{2}\right] \\
& =-\dot{F} \frac{\langle X, T\rangle_{s s}}{F}+\dot{F} \frac{\langle X, T\rangle F(K)_{s s}}{F^{2}} .
\end{aligned}
$$

Using the relation

$$
H_{s s}=\frac{1}{F^{2}}\left[F\langle X, T\rangle_{s s}-\langle X, T\rangle F(K)_{s s}\right]-\frac{2}{F} F(K)_{s} H_{s}
$$

into (3.8), we can justify equation (3.7).

Proposition 3.3. There exists a constant $C$ depending on $\gamma_{0}$ and $F$ such that

$$
\left|r_{1}(\theta, t)\right|=\left|\frac{\partial r}{\partial \theta}(\theta, t)\right| \leq C \quad \text { on } S^{1} \times[0, T)
$$

Proof. We let $C$ denote any constant depending on $\gamma_{0}$ and $F$, where $C$ may change from line to line. Applying the maximum principle to equation (3.7) immediately gives us

$$
|H| \leq C .
$$

Since

$$
H=\frac{\langle X, T\rangle}{F}=\frac{r r_{1}}{\sqrt{A} F}
$$


we find

$$
\left|r_{1}\right| \leq C \sqrt{\left(1+\frac{r_{1}^{2}}{r^{2}}\right)} F(K)
$$

At any point $(\eta, t)$ where $r_{1}(\eta, t)=\max _{\theta \in S^{1}} r_{1}(\theta, t)$, we have

$$
r_{2}(\eta, t)=0
$$

and hence

$$
K(\eta, t)=\frac{r^{2}+2 r_{1}^{2}}{A^{3 / 2}}(\eta, t)>0 .
$$

Since $F$ is decreasing, we have $F(K(\eta, t)) \leq F(0)$. By $(2.7)$ and (3.10), the proof is complete.

Since $r_{1}(\theta, t)$ is uniformly bounded on $S^{1} \times[0, T)$, we have

Corollary 3.4. The evolving curves $\gamma_{t}$ remain starshaped with respect to the origin of $\mathbb{R}^{2}$ on the time interval $[0, T)$.

\section{Second derivative estimate.}

In this section we shall derive an estimate for the second derivative of $r$, i.e. $r_{2}$, which depends on $r_{0}, T$, and $F$. The second derivative estimate comes as a consequence of the curvature estimate. We shall show that $K \geq l$ on $S^{1} \times[0, T)$, where $l$ is a constant depending on $r_{0}, T$, and $F$. This, together with (2.15), are essential for us when we want to use the results of Krylov and Safonov [15] to prove the long time existence of a solution to equation (1.2).

First, the lower bound of $r_{2}$ is an immediate consequence of (2.15). We have

Lemma 4.1. There exists a constant $C_{3}$ depending on the initial curve $\gamma_{0}$, $T$ and $F$ such that

$$
r_{2} \geq C_{3} \quad \text { on } S^{1} \times[0, T)
$$

Proof. Since $K$ is bounded above by a positive constant $C_{2}$ depending only on $\gamma_{0}$ on $S^{1} \times[0, T)$, we have

$$
r_{2} \geq \frac{r^{2}+2 r_{1}^{2}-C_{2} A^{3 / 2}}{r}
$$


The result follows from (4.2).

It is more difficult to get an upper bound of $r_{2}$. The idea is to get an upper bound of $F(K)$ first, since the evolution equation of it does not involve the uncontrolled quantity $\ddot{F}(K)$. Because we assume $\lim _{z \rightarrow-\infty} F(z)=\infty$, the fact that $F(K)$ has an upper bound will force $K$ to have a lower bound, which, in turn, implies an upper bound of $r_{2}$. We provide the details as follows. First we need

Lemma 4.2. We have

$$
\frac{\partial \Omega}{\partial \tau}=-\dot{F} \Omega_{s s}-2 \dot{F} \frac{\langle X, N\rangle_{s}}{\langle X, N\rangle} \Omega_{s}-\frac{1}{\langle X, N\rangle^{2}}\left(F^{2}+K F \dot{F}\right),
$$

where $\Omega=\frac{F}{\langle X, N\rangle}$.

Proof. It follows from (3.1) that

$$
\begin{aligned}
\frac{\partial \Omega}{\partial \tau} & =\frac{1}{\langle X, N\rangle}\left[-\dot{F} F(K)_{s s}-\dot{F} F K^{2}\right] \\
& -\frac{F}{\langle X, N\rangle^{2}}\left[-\dot{F}\langle X, N\rangle_{s s}-K^{2} \dot{F}\langle X, N\rangle+F+K \dot{F}\right] \\
& =\frac{1}{\langle X, N\rangle}\left[-\dot{F} F(K)_{s s}\right]-\frac{F}{\langle X, N\rangle^{2}}\left[-\dot{F}\langle X, N\rangle_{s s}+F+K \dot{F}\right]
\end{aligned}
$$

Substitute the relation

$$
\Omega_{s s}=\frac{1}{\langle X, N\rangle^{2}}\left[\langle X, N\rangle F(K)_{s s}-F\langle X, N\rangle_{s s}\right]-\frac{2}{\langle X, N\rangle}\langle X, N\rangle_{s} \Omega_{s}
$$

into (4.4) to get

$$
\frac{\partial \Omega}{\partial \tau}=-\dot{F}\left[\Omega_{s s}+\frac{2}{\langle X, N\rangle}\langle X, N\rangle_{s} \Omega_{s}\right]-\frac{F}{\langle X, N\rangle^{2}}[F+K \dot{F}] .
$$

The proof is done.

Lemma 4.3. There exists a constant $C_{4}$ depending on the initial curve $\gamma_{0}, T$, and $F$ such that

$$
F(K) \leq C_{4} \quad \text { on } S^{1} \times[0, T) .
$$


Proof. First note that

$$
\Omega=\frac{F}{\langle X, N\rangle}=\frac{\sqrt{A}}{r^{2}} F
$$

Using

$$
\frac{\partial}{\partial \tau}=\frac{\partial}{\partial t}+\frac{\partial \theta}{\partial \tau} \frac{\partial}{\partial \theta}, \quad \frac{\partial \theta}{\partial \tau}=-\frac{F(K)}{\sqrt{A}} \frac{r_{1}}{r}
$$

and

$$
\frac{\partial}{\partial s}=\frac{1}{\sqrt{A}} \frac{\partial}{\partial \theta}
$$

we can convert equation (4.3) into

$$
\begin{aligned}
\frac{\partial}{\partial t}\left(\frac{A^{1 / 2}}{r^{2}} F\right) & =-\frac{\dot{F}}{A}\left(\frac{A^{1 / 2}}{r^{2}} F\right)^{\prime \prime}-4 \frac{\dot{F}}{A} \frac{r_{1}}{r}\left(\frac{A^{1 / 2}}{r^{2}} F\right)^{\prime}+\frac{3}{2} \frac{\dot{F}}{A^{2}} A^{\prime}\left(\frac{A^{1 / 2}}{r^{2}} F\right)^{\prime} \\
+ & \frac{F}{A^{1 / 2}} \frac{r_{1}}{r}\left(\frac{A^{1 / 2}}{r^{2}} F\right)^{\prime}-\frac{A}{r^{4}} F(K \dot{F}+F) .
\end{aligned}
$$

on $S^{1} \times[0, T)$. Let $C_{5}$ be a constant depending on $r_{0}$ and $F$ such that

$$
\frac{\sqrt{A}}{r^{2}}=\left(\frac{1}{r^{2}}+\frac{r_{1}^{2}}{r^{4}}\right)^{1 / 2} \leq C_{5} \quad \text { on } S^{1} \times[0, T)
$$

and let $\left(\frac{A^{1 / 2}}{r^{2}} F\right)_{\max }(t)=\max _{\theta \in S^{1}}\left(\frac{A^{1 / 2}}{r^{2}} F\right)(\theta, t)$. At any point $(p, t)$ where $\left(\frac{A^{1 / 2}}{r^{2}} F\right)(p, t)=\max _{\theta \in S^{1}}\left(\frac{A^{1 / 2}}{r^{2}} F\right)(\theta, t)$, if $\left(\frac{A^{1 / 2}}{r^{2}} F\right)_{\max }(t) \geq C_{5} F(0)$, we must have $K(p, t) \leq 0$. Therefore $\partial_{t}\left(\frac{A^{1 / 2}}{r^{2}} F\right) \leq 0$ at $(p, t)$ by (4.7). Because we know

$$
\frac{d^{+}}{d t}\left(\frac{A^{1 / 2}}{r^{2}} F\right)_{\max }(t) \leq \sup \left\{\partial_{t}\left(\frac{A^{1 / 2}}{r^{2}} F\right)(\xi, t), \xi \in E(t)\right\},
$$

where $E(t)=\left\{\theta \in S^{1},\left(\frac{A^{1 / 2}}{r^{2}} F\right)(\theta, t)=\left(\frac{A^{1 / 2}}{r^{2}} F\right)_{\max }(t)\right\}$. We deduce that

$$
\frac{d^{+}}{d t}\left(\frac{A^{1 / 2}}{r^{2}} F\right)_{\max }(t) \leq 0
$$

whenever $\left(\frac{A^{1 / 2}}{r^{2}} F\right)_{\max }(t) \geq C_{5} F(0)$. Hamilton's maximum principle guarantees that

$$
\left(\frac{A^{1 / 2}}{r^{2}} F\right)_{\max }(t) \leq \max \left\{\left(\frac{A^{1 / 2}}{r^{2}} F\right)_{\max }(0), C_{5} F(0)\right\}
$$


for all $t \in[0, T)$. The proof of (4.5) is complete.

Remark: Note that $\frac{A^{1 / 2}}{r^{2}} F=\frac{\partial_{t} r}{r}$.

Proposition 4.4. There exists a constant $C_{6}$ depending on the initial curve $\gamma_{0}, T$ and $F$ such that

$$
\left|r_{2}(\theta, t)\right| \leq C_{6} \quad \text { on } S^{1} \times[0, T) .
$$

Proof. Since $F(K) \leq C_{4}$ and $\lim _{z \rightarrow-\infty} F(z)=\infty$, we have $K \geq C_{7}$ for some constant $C_{7}$. This implies that

$$
r_{2} \leq \frac{r^{2}+2 r_{1}^{2}-C_{7} A^{3 / 2}}{r} \text { on } S^{1} \times[0, T) .
$$

Combined with (4.1), the proof is done.

\section{Long time existence.}

Proposition 5.1. (Long time existence) There exists a unique smooth solution $r(\theta, t)$ to equation (1.2) on $S^{1} \times[0, \infty)$.

Proof. In the following discussions, we shall restrict the domain of all quantities to $S^{1} \times[\epsilon, T)$ where $\epsilon$ is some fixed positive constant. Also, we let $\hat{C}$ denote any constant depending on $\epsilon, T, F$, and $r_{0}$, where $\hat{C}$ may change from line to line. Recall the following three equations

$$
\begin{aligned}
\partial_{t} r_{1} & =-\frac{\dot{F}}{A} r_{3}+\left[\frac{F}{A^{1 / 2}}+\frac{\dot{F}}{A^{2}}\left(3 r^{2}-3 r_{1}^{2}+3 r r_{2}\right)\right] \frac{r_{1}}{r} r_{2} \\
& -\frac{F}{A^{1 / 2}} \frac{r_{1}^{3}}{r^{2}}-\frac{\dot{F}}{A^{2}}\left(4 r_{1}^{3}+r^{2} r_{1}\right),
\end{aligned}
$$

$$
\partial_{t} F(K)=-\frac{\dot{F}}{A} F(K)^{\prime \prime}+\frac{F}{A^{1 / 2}} \frac{r_{1}}{r} F(K)^{\prime}+\frac{A^{\prime}}{2 A^{2}} \dot{F} F(K)^{\prime}-\dot{F} K^{2} F(K)
$$

and

$$
\frac{\partial r}{\partial t}=\frac{A^{1 / 2}}{r} F=-\frac{\dot{F}}{A} r_{2}+2 \frac{\dot{F}}{A} \frac{r_{1}^{2}}{r}+\frac{\dot{F}}{A} r+\frac{A^{1 / 2}}{r}(F-K \dot{F}) .
$$


Moreover, it is not difficult to compute

$$
\begin{aligned}
\partial_{t} \Phi & =-\frac{\dot{F}}{A} \Phi^{\prime \prime}-2 \frac{\dot{F}}{A} \frac{r_{1}}{r} \Phi^{\prime}+\frac{3}{2} \frac{\dot{F}}{A^{2}} A^{\prime} \Phi^{\prime}+\frac{F}{A^{1 / 2}} \frac{r_{1}}{r} \Phi^{\prime} \\
& +\frac{F \dot{F}}{A^{5 / 2}} \frac{1}{r^{3}}\left[\left(r^{6}-3 r^{2} r_{1}^{4}-2 r_{1}^{6}\right)-\left(2 r^{4}+r^{2} r_{1}^{2}-r_{1}^{4}\right) \cdot K A^{3 / 2}\right] \\
& -\frac{r_{1}^{2}}{r^{3}} F(K)^{2}
\end{aligned}
$$

where $\Phi=\frac{A^{1 / 2}}{r} F(K)=\frac{\partial r}{\partial t}$. Since we have deduced

$$
|r|,\left|r_{1}\right|,\left|r_{2}\right|,|K|,|F(K)|,|\dot{F}(K)| \leq \hat{C},
$$

we can apply Theorem 4.2 in Krylov-Safonov [15] to equations (5.1), (5.2), (5.3) and (5.4) to yield

$$
\begin{aligned}
& \|r\|_{C^{0, \alpha}\left(S^{1} \times[\epsilon, T)\right)},\left\|r_{1}\right\|_{C^{0, \alpha}\left(S^{1} \times[\epsilon, T)\right)} \leq \hat{C} \\
& \left\|\frac{\partial r}{\partial t}\right\|_{C^{0, \alpha}\left(S^{1} \times[\epsilon, T)\right)},\|F(K)\|_{C^{0, \alpha}\left(S^{1} \times[\epsilon, T)\right)} \leq \hat{C}
\end{aligned}
$$

for some constant $\alpha \in(0,1)$. Because $F$ is smooth and decreasing, we clearly have

$$
\|K\|_{C^{0, \alpha}\left(S^{1} \times[\epsilon, T)\right)} \leq \hat{C} .
$$

Using

$$
r_{2}=\frac{r^{2}+2 r_{1}^{2}-K A^{3 / 2}}{r}
$$

and (5.6), (5.7), we finally get

$$
\left\|\frac{\partial r}{\partial t}\right\|_{C^{0, \alpha}\left(S^{1} \times[\epsilon, T)\right)}+\left\|r_{2}\right\|_{C^{0, \alpha}\left(S^{1} \times[\epsilon, T)\right)} \leq \hat{C} .
$$

Estimate (5.8) is good enough for us to apply the standard parabolic theory to get the $C^{k, \alpha}$ bounds of $r$ for any positive integer $k$. The proof is done.

Remark: Alternatively, we can also show that all of the derivatives of the curvature $K$ with respect to the arc length $s$ are bounded as long as $r$ is finite. This will allow us to get long time existence without using the theorem of Krylov and Safonov. See also Chow-Tsai [5]. 


\section{Rescaling and convergence.}

Since we have long time existence, we are ready to investigate the asymptotic behavior of the solution. We shall see that the quantitative behavior of $r(\theta, t)$ is the same as some solution $R(t)$ to the o.d.e.

$$
\frac{d}{d t} R(t)=F\left(\frac{1}{R}\right)
$$

First, we have

Lemma 6.1. There exists a solution $R(t)$ to the o.d.e. (6.1) such that

$$
r_{\min }(t) \leq R(t) \leq r_{\max }(t)
$$

for all $t \in[0, \infty)$.

Proof. First note that if $r_{\max }(t)=r(p, t)$ for some $p \in S^{1}$, then

$$
\begin{aligned}
\frac{\partial r}{\partial t}(p, t) & =\frac{A^{1 / 2}}{r} F\left(\frac{r^{2}+2 r_{1}^{2}-r r_{2}}{A^{3 / 2}}\right)(p, t) \\
& =F\left(\frac{r^{2}-r r_{2}}{r^{3}}\right)(p, t) \leq F\left(\frac{1}{r_{\max }(t)}\right) .
\end{aligned}
$$

Similarly, if $r_{\min }(t)=r(q, t)$ for some $q \in S^{1}$, we have

$$
\frac{\partial r}{\partial t}(q, t) \geq F\left(\frac{1}{r_{\min }(t)}\right) .
$$

The rest of the proof goes exactly the same as in Lemma 9 in Chow-Tsai [5]. We omit it.

Now we will choose one $R(t)$ satisfying (6.2) and use it to rescale the solution $r(\theta, t)$. Define the rescaled solution $\tilde{r}(\theta, t)$ as

$$
\tilde{r}(\theta, t)=\frac{r(\theta, t)}{R(t)} .
$$

Lemma 6.2. We have

$$
|\tilde{r}(\theta, t)-1| \leq \frac{2 \pi C}{R(t)}
$$


and

$$
\left|\frac{\partial \tilde{r}}{\partial \theta}(\theta, t)\right| \leq \frac{C}{R(t)}
$$

for all $(\theta, t) \in[0, \infty)$, where $C$ is the constant in (3.9).

Proof. By Proposition 3.3, we have

$$
|\tilde{r}(\theta, t)-1|=\left|\frac{r(\theta, t)-R(t)}{R(t)}\right| \leq \frac{r_{\max }(t)-r_{\min }(t)}{R(t)} \leq \frac{2 \pi C}{R(t)} .
$$

Equation (6.4) is also a consequence of Proposition 3.3.

The proof of Theorem 1.1 is now complete.

Before we end this section, we would like to say something about the asymptotic behavior of the curvature. Since we can not obtain a good estimate on the second derivative of $r$, we are not able to get sharp control of the curvature. However, we can deduce the following rough estimate.

Lemma 6.3. We have

(i) $\lim _{t \rightarrow \infty} \sup K_{\max }(t)=0$.

(ii) There exists a positive constant $d$ depending on $\gamma_{0}$ and $F$ such that

$$
K(\theta, t) \geq-d,
$$

for all $(\theta, t) \in S^{1} \times[0, \infty)$.

Proof. (i) Recall equation (2.10), which is

$$
\partial_{t} K=-\frac{\dot{F}}{A} K^{\prime \prime}+\frac{F}{A^{1 / 2}} \frac{r_{1}}{r} K^{\prime}+\frac{A^{\prime}}{2 A^{2}} \dot{F} K^{\prime}-\frac{\ddot{F}}{A} K^{\prime 2}-F K^{2} .
$$

In view of the comparison principle, we have

$$
0 \leq K_{\max }(t) \leq \varphi(t)
$$

on $[0, \infty)$, where $\varphi(t)$ is the solution to the o.d.e.

$$
\begin{aligned}
& \frac{d \varphi}{d t}=-F(\varphi) \varphi^{2} \\
& \varphi(0)=K_{\max }(0)>0
\end{aligned}
$$


It is evident that $\lim _{t \rightarrow \infty} \varphi(t)=0$. (i) is proved.

(ii) We remember the equation

$$
\begin{aligned}
\partial_{t} \Phi & =-\frac{\dot{F}}{A} \Phi^{\prime \prime}-2 \frac{\dot{F}}{A} \frac{r_{1}}{r} \Phi^{\prime}+\frac{3}{2} \frac{\dot{F}}{A^{2}} A^{\prime} \Phi^{\prime}+\frac{F}{A^{1 / 2}} \frac{r_{1}}{r} \Phi^{\prime} \\
& +\frac{F \dot{F}}{A^{5 / 2}} \frac{1}{r^{3}}\left[\left(r^{6}-3 r^{2} r_{1}^{4}-2 r_{1}^{6}\right)-\left(2 r^{4}+r^{2} r_{1}^{2}-r_{1}^{4}\right) \cdot K A^{3 / 2}\right] \\
& -\frac{r_{1}^{2}}{r^{3}} F(K)^{2},
\end{aligned}
$$

where $\Phi=\frac{A^{1 / 2}}{r} F(K)$. For $t$ large enough, say $t \geq T^{\prime}$ for some $T^{\prime}>0$, we have

$$
\begin{aligned}
\partial_{t} \Phi & \leq-\frac{\dot{F}}{A} \Phi^{\prime \prime}-2 \frac{\dot{F}}{A} \frac{r_{1}}{r} \Phi^{\prime}+\frac{3}{2} \frac{\dot{F}}{A^{2}} A^{\prime} \Phi^{\prime}+\frac{F}{A^{1 / 2}} \frac{r_{1}}{r} \Phi^{\prime} \\
& -\frac{F \dot{F}}{A^{5 / 2}} \frac{1}{r^{3}}\left(2 r^{4}+r^{2} r_{1}^{2}-r_{1}^{4}\right) \cdot K A^{3 / 2}
\end{aligned}
$$

and $\left(2 r^{4}+r^{2} r_{1}^{2}-r_{1}^{4}\right)>0$ on $S^{1} \times\left[T^{\prime}, \infty\right)$. Now let $C_{8}$ be a constant such that $\frac{A^{1 / 2}}{r} \leq C_{8}$ on $S^{1} \times\left[T^{\prime}, \infty\right)$. Again at any point $(p, t)$ where $\left(\frac{A^{1 / 2}}{r} F\right)(p, t)=$ $\max _{\theta \in S^{1}}\left(\frac{A^{1 / 2}}{r} F\right)(\theta, t)$ and $t>T^{\prime}$, if $\left(\frac{A^{1 / 2}}{r} F\right)_{\max }(t) \geq C_{8} F(0)$, we must have $K(p, t) \leq 0$. Therefore $\partial_{t}\left(\frac{A^{1 / 2}}{r} F\right) \leq 0$ at $(p, t)$ by $(6.5)$. Hence

$$
\left(\frac{A^{1 / 2}}{r} F\right)_{\max }(t) \leq \max \left\{\left(\frac{A^{1 / 2}}{r} F\right)_{\max }\left(T^{\prime}\right), C_{8} F(0)\right\},
$$

on $S^{1} \times\left[T^{\prime}, \infty\right)$. Since we assume $\lim F(z)=\infty$, the $z \rightarrow-\infty$ proof of (ii) is done.

Acknowledgement. I would like to thank my advisor Ben Chow for posing this problem to me. His patient guidance and constant encouragement have been very influential through the years. Thanks also go to Mr. Lii-Perng Liou for many helpful discussions and the referee for some suggestions. 


\section{References.}

[1] Andrews, B. (1993) thesis, Evolving convex hypersurfaces, The Australian National University.

[2] Chow, B. (1995) Geometric aspects of Aleksandrov reflection and gradient estimates for parabolic equations, to appear in Comm. Anal. and Geom. $1996 / 1997$.

[3] Chow, B., Gulliver, R. (1994) Aleksandrov reflection and nonlinear evolution equations, I: The $n$-sphere and $n$-ball, to appear.

[4] Chow, B., Gulliver, R. (1995) Aleksandrov reflection and geometric evolution of embedded hypersurfaces, in preparation.

[5] Chow, B., Tsai, D.H. (1994) Geometric expansion of convex plane curves, to appear in J. Diff. Geom. 1996.

[6] Chow, B., Tsai, D.H. (1995) Expansion of convex hypersurfaces by nonhomogeneous functions of curvature, in preparation.

[7] Chow, B., Liou, L.P., Tsai, D.H. (1995) On the nonlinear parabolic equation $u_{t}=F(\Delta u+n u)$ on $S^{n}$, to appear in Comm. Anal. and Geom. 1996/1997.

[8] Chow, B., Liou, L.P., Tsai, D.H. (1995) Expansion of embedded plane curves with turning angle greater than $-\pi$, to appear in Inventiones Mathematicae 1996.

[9] Gage, M., Hamilton, R. S. (1986) The heat equation shrinking convex plane curves, J. Diff. Geom. 23, 69-96.

[10] Gerhardt, C. (1990) Flow of nonconvex hypersurfaces into spheres, J. Diff. Geom. 32, 299-314.

[11] Hamilton, R.S. (1982) Three-manifolds with positive Ricci curvature, J. Diff. Geom. 17, 255-306.

[12] Hamilton, R.S. (1986) Four-manifolds with positive curvature operator, J. Diff. Geom. 24, 153-179.

[13] Huisken, G. (1984) Flow by mean curvature of convex hypersurfaces into spheres, J. Diff. Geom. 20, 237-268.

[14] Huisken, G. (1988) On the expansion of convex hypersurfaces by the inverse of symmetric functions, preprint. 
[15] Krylov, N.V., Safonov, M.V. (1980) Certain properties of parabolic equations with measurable coefficients, Izv. Akad. Nauk SSSR Ser. Mat. 40, 161-175, English tsansl., Math. USSR Izv. 16 (1981) 151-164.

[16] Tsai, D.H. (1995) Geometric expansion of immersed convex plane curves, in preparation.

[17] Tso, K. (1985) Deforming a hypersurface by its Gauss-Kronecker curvature, Comm. Pure and Appl. Math. 38, 867-882.

[18] Urbas, J.I.E. (1991) An expansion of convex hypersurfaces, J. Diff. Geom. 33, 91-125; (1992) Correction to, ibid. 35, 763-765.

[19] Urbas, J.I.E. (1990) On the expansion of starshaped hypersurfaces by symmetric functions of their principal curvatures, Math. Z. 205, 355-372.

RECEIVED FEBRUARY 28, 1995.

SCHOOL OF MATHEMATICS

UNIVERSITY OF MINNESOTA

MINNEAPOLIS, MN 55455 